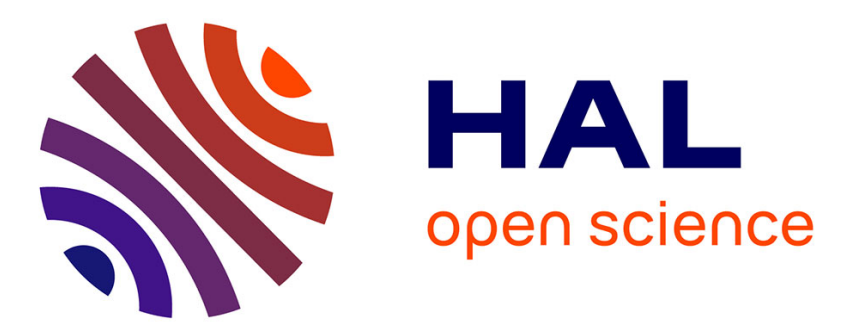

\title{
Distributed Control of coordinated path tracking for networked nonholonomic mobile vehicles
}

\author{
Zhang Qin, Lionel Lapierre, Xianbo Xiang
}

\section{To cite this version:}

Zhang Qin, Lionel Lapierre, Xianbo Xiang. Distributed Control of coordinated path tracking for networked nonholonomic mobile vehicles. IEEE Transactions on Industrial Informatics, 2012, pp.1. 10.1109/TII.2012.2219541 . hal-00733822

\section{HAL Id: hal-00733822 \\ https://hal.science/hal-00733822}

Submitted on 19 Sep 2012

HAL is a multi-disciplinary open access archive for the deposit and dissemination of scientific research documents, whether they are published or not. The documents may come from teaching and research institutions in France or abroad, or from public or private research centers.
L'archive ouverte pluridisciplinaire HAL, est destinée au dépôt et à la diffusion de documents scientifiques de niveau recherche, publiés ou non, émanant des établissements d'enseignement et de recherche français ou étrangers, des laboratoires publics ou privés. 


\section{Distributed control of coordinated path tracking for networked nonholonomic mobile vehicles}

\begin{abstract}
This paper addresses the problem of coordinated path tracking for networked nonholonomic mobile vehicles, while building and keeping a desired formation. The control laws proposed are categorized into two envelopes by integrating individual path tracking and global virtual structure approaches. One is steering individual vehicles to track virtual vehicles moving along predefined paths, generated by a formation reference vehicle (FRV) of a time-varying desired virtual structure. The other is ensuring paths to be well tracked in order to build a geometric formation, through the distributed feedback law for path parameters related to the virtual vehicles, such that the physical vehicles are on the desired placements of the formation structure. Within this framework, geometric path tracking is achieved via nonlinear control theory, where an approaching angle is injected as a heading guidance design. The distributed feedback law is analyzed under communication constraints using algebraic graph theory. It is formally shown that the path tracking error of each vehicle is reduced to zero, and vehicles in the networked team globally asymptotically converge to a desired formation with equal path parameters. Simulation results illustrate the effectiveness of the proposed control design.
\end{abstract}

Index Terms-Coordinated path tracking, nonholonomic mobile vehicle, distributed control, formation reference vehicle.

\section{INTRODUCTION}

Compared with a single vehicle, a team of networked autonomous vehicles dealing with tasks offers additional advantages, in terms of flexibility, robustness and efficiency. $\mathrm{Nu}-$ merous applications related to networked vehicles, including air, land, marine vehicles and in combinations thereof [1], are presently operational in industrial, scientific and military fields and more ambitious applications are in engineering development [2]. In order to meet various goals of different applications, autonomous vehicles must be equipped with control systems to steer them to achieve various motion tasks, and there is considerable interest in the development of advanced methods for coordinated and cooperative motion control of multiple vehicles [3], [4]. Coordinated formation control has been one of the hot topics in networked multi-vehicle systems, and research work in this field can be classified into four categories, i.e. leader-follower, behavioral, virtual structure and artificial potential approaches.

In the leader-follower approach, the basic idea is that the leaders track predefined reference trajectories, and the followers track transformed versions of the states of the leaders according to predefined schemes (e.g., assigned formation configuration with the leaders) [5]. In [6], [7], the leaderfollower based formation control is applied to multiple mobile vehicles depending on relative orientations/distances as so called $l-\psi / l-l$ control. In [8], autonomous surface vessels are synchronized through a leader-follower synchronization output feedback control scheme to implement a replenishment problem. An advantage of the leader-follower approach is that it is easy to understand and implement, since the coordinated team members only need to maneuver according to the leader. However, there is no explicit feedback from the followers to the leader, and the failure of the leader leads to the failure of the whole formation team.

In [9], the behavior based approach for a mobile robotic team is reported, where move-to-goal, avoid-static obstacle, avoid-robot and formation maintenance behaviors, are integrated through suitable weight coefficients in terms of relative priorities of behaviors. In [10], it provides a clear example of a behavioral approach for formation flight. In [11], the nullspace-based (NSB) behavioral control of a fleet of marine surface vessels is presented. Behavior-based approaches give the system the autonomy to operate in an unknown or dynamically changing environment, by defining and integrating different behaviors dedicated to specified sub-tasks. However, the group behavior cannot be explicitly defined, and it is difficult to analyze the approach mathematically.

In the framework of virtual structure approach, a group of mobile robots achieve high precision formation control where each member in the formation is taken as a node in a rigid geometric structure [12]. Some similar ideas are given based on the perceptive reference frame [13], the virtual leader generating the virtual rigid body [14], and the formation reference point [15] respectively. In [16], a specific form of formation feedback is introduced in the virtual structure based control, for a team of mobile robots. The main advantage of the virtual structure approach is that it is fairly easy to prescribe the coordinated behavior for the whole formation group, and add a type of robustness to formation through the use of formation feedback. Thus, the formation can be maintained very well while maneuvering. The disadvantage is that requiring the formation to act as a rigid virtual structure limits the class of potential applications.

In [17], [18], artificial potentials generate interaction forces between neighboring vehicles to enforce a desired inter-vehicle spacing. In [19], artificial potentials are used to avoid obstacles and guarantee collision avoidance between mobile agents, to split and merge subgroups, and to perform squeezing maneuvers for a large number of agents. In [20], a regionbased controller for a swarm of fully actuated mobile robots is proposed by using potential energy functions. The artificial potential approach is suitable to control a large group of vehicles in a loose formation pattern through the attractive and repulsive forces. However, it is difficult to build a desired rigid shape for networked multi-vehicle systems by means of artificial potential forces. Moreover, the local minimum problem hinders the applications of artificial potentials.

This paper is aimed at designing coordinated path tracking control laws in a flexible and distributed manner for a team of networked nonholonomic mobile vehicles under inter-vehicle 
communication constraints. The main contributions of this paper are stated through addressing three challenges upon reviewing solutions in the related literatures. (1) The first challenge is that the nonholonomic characteristics render the coordinated path tracking control design to be more difficult, compared with the consensus algorithm for agents with simple dynamics, for instance, a first or second order integrator dynamics in [21], [22], [23], [24]. Although passivity-based designs are proposed to synchronize marine vehicles following predefined paths with complex dynamics in [25], the fully actuated model of marine vehicles do not have the lateral zerospeed constraint imposed on nonholonomic mobile vehicles. Herein, Lyapunov based design and backstepping techniques, along with dedicated approaching angle guidance, are adopted to address the nonholonomic constraints. (2) The second challenge is that the selection of path variables for coordinated path following is critical to get flexible coordination. In [26], [27], [28], the distance along a path/arc (e.g. curvilinear abscissa) is chosen as a path variable to build a desired geometric formation, which is only suitable for shifted or parallel paths. In [29], normalized lengths of the curvilinear abscissas are adopted and transformed coordination error dynamics are further required, which renders an indirect way for coordinated path following control design. In this paper, a modified virtual structure approach is used to get flexible path variables related to the virtual formation reference vehicle. This flexibility also enables construction of time-varying formations in terms of general curved paths. (3) The third challenge is that the inter-vehicle communication constraints exist while a team of networked vehicles follow predefined paths in a coordinated manner. In [30], [31], coordinated path following control is also partially solved based on the virtual structure method. However, all-to-all communications are considered and centralized control is proposed in these literatures. In this paper, graph theory is resorted to achieve distributed control design under inter-vehicle communication constraints. On the other hand, the path following controllers adopted in [26], [29], [32], [33] account for communication constraints, and introduce an additional control degree of freedom to the virtual target. The tradeoff is that only spatial convergence to the path is guaranteed. However, the distributed path tracking controllers adopted in this paper can also guarantee temporal convergence through time-like path variables related to the virtual vehicles moving along the desired paths [34].

The rest of the paper is organized as follows. The problem statement is presented in the next section. In section III, the individual path tracking control with the injection of approaching angle guidance is derived, and then the coordinated path tracking control under the constraints of the communication network is presented, in both kinematics and dynamics stages. Numerical simulations of two types of circle formation are illustrated in Section IV. Section V concludes the paper. Proofs of the main result is given in Appendix.

Notation: The notations used in this paper are standard. The time derivatives of $\tau_{i}(t)$ are denoted $\dot{\tau}_{i}, \ddot{\tau}_{i}, \tau_{i}^{(3)}, \ldots, \tau_{i}^{(n)}$, while a superscript denotes partial differentiation: $x_{r i}^{\tau_{i}}\left(\tau_{i}\right)=\partial x_{r i}\left(\tau_{i}\right) / \partial \tau_{i}, \quad x_{r i}^{\tau_{i}^{2}}\left(\tau_{i}\right)=\partial^{2}\left(x_{r i}\left(\tau_{i}\right)\right) / \partial \tau_{i}^{2}, \quad$ and $x_{r i}^{\tau_{i}^{n}}\left(\tau_{i}\right)=\partial^{n}\left(x_{r i}\left(\tau_{i}\right)\right) / \partial \tau_{i}^{n}$. The Euclidean vector norm is $\|x\|:=\sqrt{x^{T} x}$.

\section{Problem StATEMENT}

In this paper, we consider a networked team of $n \geq 2$ nonholonomic unicycle-type mobile vehicles, labeled 1 through $n$, tracking a set of paths $\Gamma_{i}\left(\tau_{i}\right), i=1, \ldots, n$, while attaining a desired inter-vehicle formation.

\section{A. Model of nonholonomic mobile vehicle}

In Fig. 1, unicycle-type mobile vehicle $i$ is required to track a predefined spatial path $\Gamma_{i}\left(\tau_{i}\right)$, parameterized by a scalar variable $\tau_{i}$. Each vehicle $i \in\{1, \ldots, n\}$ in the team of $n$ vehicles has two identical parallel, non-deformable rear wheels and a passive front wheel.

The coordinates of the $i$-th vehicle in the inertial frame $\{I\}$ are $p_{i}=\left(x_{i}, y_{i}, \psi_{i}\right)^{T}$, where $\left(x_{i}, y_{i}\right)^{T}$ denotes the position of the wheel axis center and $\psi_{i}$ denotes the vehicle orientation with respect to the $X$-axis. Let $u_{i}$ and $\omega_{i}$ denote the linear and angular velocity of the vehicle in the body frame $\left\{B_{i}\right\}$ with respect to $\{I\}$ respectively. The kinematic model for the $i$-th unicycle-type nonholonomic mobile vehicle is defined as:

$$
\left[\begin{array}{c}
\dot{x}_{i} \\
\dot{y}_{i} \\
\dot{\psi}_{i}
\end{array}\right]=\left[\begin{array}{cc}
\cos \psi_{i} & 0 \\
\sin \psi_{i} & 0 \\
0 & 1
\end{array}\right]\left[\begin{array}{l}
u_{i} \\
\omega_{i}
\end{array}\right]
$$

The control input vector $\left(F_{i}, N_{i}\right)^{T}$ provides the forward force $F_{i}$ and angular torque $N_{i}$ applied on the center of mass of the vehicle. The vehicle mass and moment of inertia are denoted as $m_{i}$ and $I_{i}$ respectively. It is assumed that the plane of each wheel of the vehicle is perpendicular to the ground and the contact between the wheels and the ground is pure rolling and nonslipping. It is further assumed that the masses and inertias of the wheels are negligible and the center of mass of the mobile vehicle is located in the middle of the axis connecting the rear wheels [35], [36]. Thus, the dynamic model of the unicycle-type autonomous vehicle is obtained by augmenting (1) with the equations

$$
\left[\begin{array}{c}
F_{i} \\
N_{i}
\end{array}\right]=\left[\begin{array}{cc}
m_{i} & 0 \\
0 & I_{i}
\end{array}\right]\left[\begin{array}{c}
\dot{u}_{i} \\
\dot{\omega}_{i}
\end{array}\right]
$$

Actually, a unicycle-type vehicle suffers from the first-order nonholonomic constraint (also called the lateral zero-speed constraint), such that its linear velocity is always aligned with the longitudinal axis due to $\dot{y}_{i} \cos \psi_{i}-\dot{x}_{i} \sin \psi_{i}=0$ [37].

\section{B. Path formulation}

For a team of $n$ vehicles, the conventional virtual structure approach is modified to generate $n$ prescribed reference paths, which are suitable for coordinated path tracking control in a formation. The basic idea is to use a virtual moving vehicle called the formation reference vehicle (FRV), which defines the center of a virtual structure, to represent the whole formation as one moving virtual structure. In Fig. 2, the FRV moves along a given "baseline" path $\Gamma_{0}\left(\tau_{0}\right)=\operatorname{col}\left(p_{r 0}\left(\tau_{0}\right), \psi_{r 0}\left(\tau_{0}\right)\right)$ in horizontal plane, where $p_{r 0}\left(\tau_{0}\right)=\left[x_{r 0}\left(\tau_{0}\right), y_{r 0}\left(\tau_{0}\right)\right]^{T}$ is the 


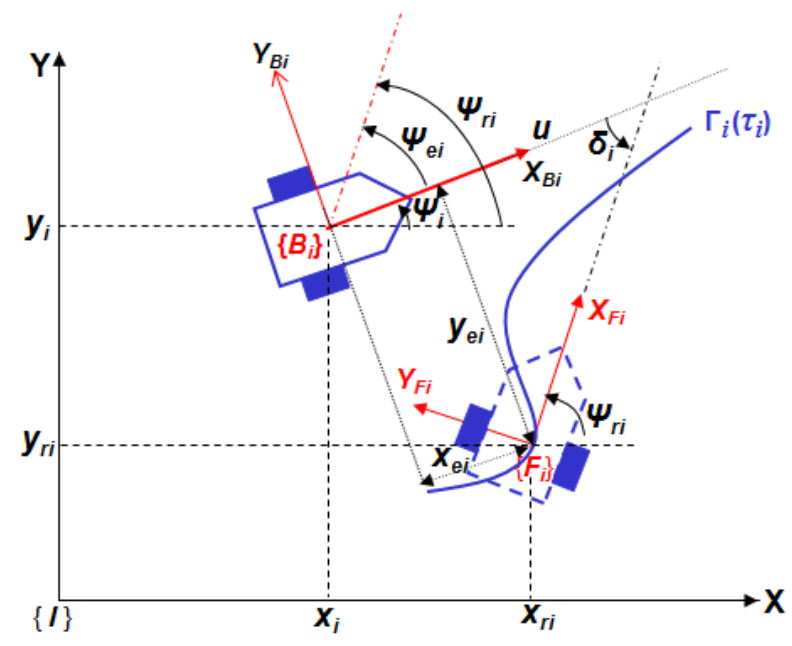

Fig. 1. The $i$-th nonholonomic unicycle-type vehicle tracks the $i$-th path

desired position of the FRV in the inertial frame, and $\psi_{r 0}\left(\tau_{0}\right)$ is the desired orientation along the baseline. Attached to the FRV, the Frenet-Serret frame $\{\mathrm{F}\}$ is built by choosing the tangent vector along the baseline as $X_{F}$ and the principal normal vector as $Y_{F}$ [38]. While the FRV evolves with a desired speed assignment, a set of $n$ paths can be generated by introducing $n$ offset vectors $l_{i}(i=1, \ldots, n)$ relative to the origin of $\{F\}$, given as $\Gamma_{i}\left(\tau_{i}\right)=\operatorname{col}\left(p_{r i}\left(\tau_{i}\right), \psi_{r i}\left(\tau_{i}\right)\right)$ such that

$$
\Gamma_{i}\left(\tau_{i}\right)=\left[\begin{array}{c}
p_{r 0}\left(\tau_{i}\right)+R_{F}^{I}\left(\psi_{r 0}\left(\tau_{i}\right)\right) l_{i}\left(x_{r i}\left(\tau_{i}\right), y_{r i}\left(\tau_{i}\right)\right) \\
\operatorname{atan} 2\left(y_{r i}^{\tau_{i}}\left(\tau_{i}\right) / x_{r i}^{\tau_{i}}\left(\tau_{i}\right)\right)
\end{array}\right]
$$

where $\operatorname{atan} 2(\cdot)$ function is used in computer simulation for implementing $\arctan (\cdot)$ with correct quadrant mapping.

The rotation matrix $R\left(\psi_{r 0}\left(\tau_{i}\right)\right)$ from the Frenet-Serret frame $\{F\}$ to the inertial frame $\{I\}$, is given as

$$
R_{F}^{I}\left(\psi_{r 0}\left(\tau_{i}\right)\right)=\left[\begin{array}{cc}
\cos \left(\psi_{r 0}\left(\tau_{i}\right)\right) & -\sin \left(\psi_{r 0}\left(\tau_{i}\right)\right) \\
\sin \left(\psi_{r 0}\left(\tau_{i}\right)\right) & \cos \left(\psi_{r 0}\left(\tau_{i}\right)\right)
\end{array}\right]
$$

where $\psi_{r 0}\left(\tau_{i}\right)=\operatorname{atan} 2\left(y_{r 0}^{\tau_{i}}\left(\tau_{i}\right) / x_{r 0}^{\tau_{i}}\left(\tau_{i}\right)\right)$.

The offset vector $l_{i}$ is constructed as

$$
l_{i}\left(x_{r i}\left(\tau_{i}\right), y_{r i}\left(\tau_{i}\right)\right)=\left[\begin{array}{l}
f_{x i}\left(x_{r i}\left(\tau_{i}\right), y_{r i}\left(\tau_{i}\right)\right) \\
f_{y i}\left(x_{r i}\left(\tau_{i}\right), y_{r i}\left(\tau_{i}\right)\right)
\end{array}\right]
$$

where $f_{x i}(\cdot)$ and $f_{y i}(\cdot)$ are the offset functions related to different geometric formation constraints, such as "in-line", "triangle" or "polygonal" formation pattern, etc.

\section{Remark:}

- The baseline is not limited by the straightline in Fig. 2 , and it can be any feasible curved path, where the Frenet-Serret frame is used as a main tool in differential geometry to build and represent general curves.

- The offset vector is constant in conventional virtual structure approaches, which results in fixed formation shape. In contrast, the offset functions $f_{x i}(\cdot), f_{y i}(\cdot)$ in the offset vector (4) may be time-varying to allow for timevarying formation patterns.

- The proposed virtual-structure concept can be extended to a dynamic virtual structure with rotation and scaling. In this case, the paths can be defined as follows:

$$
p_{r i}\left(\tau_{i}\right)=p_{r 0}\left(\tau_{i}\right)+R S R_{F}^{I}\left(\psi_{r 0}\left(\tau_{i}\right)\right) l_{i}\left(x_{r i}\left(\tau_{i}\right), y_{r i}\left(\tau_{i}\right)\right)
$$

The rotation matrix is

$$
R=\left[\begin{array}{cc}
\cos \psi(t) & \sin \psi(t) \\
-\sin \psi(t) & \cos \psi(t)
\end{array}\right]
$$

where $\psi(t)$ is an angle of rotation.

The scaling matrix is

$$
S=\left[\begin{array}{cc}
s_{1}(t) & 0 \\
0 & s_{2}(t)
\end{array}\right]
$$

where $s_{1}(t)$ and $s_{2}(t)$ are scaling factors. This means that the desired virtual structure shrinks whenever the scaling factor decreases, and expands if the scaling factor increases.

\section{Control objective}

In Fig. 2, as the FRV moves along the baseline $\Gamma_{0}\left(\tau_{0}\right)$ with a desired time evolving law $\dot{\tau}_{0}(t)$, there is a corresponding virtual vehicle moving along the designated path $\Gamma_{i}\left(\tau_{i}\right)$ with a timing law $\dot{\tau}_{i}(t)$. Obviously, if all the path parameters are synchronized, i.e., $\tau_{i}=\tau_{j}, i \neq j$, the virtual vehicle member $i$ will be in the desired place-holder of the geometric formation. As the $i$-th virtual vehicle will follow the prescribed path $\Gamma_{i}\left(\tau_{i}\right)$, the remaining requirement of coordinated path tracking is to force the $i$-th physical vehicle to track the corresponding virtual vehicle on $\Gamma_{i}\left(\tau_{i}\right)$ so that the desired formation can be constructed. Hence, for the problem of coordinated path tracking for a team of networked vehicles shown in Fig. 2, there are two assignments assembled in the sense that:

1) Path tracking assignment:

ensures each individual physical vehicle converges to the corresponding virtual vehicle, and moves along the path $\Gamma_{i}\left(\tau_{i}\right)$ with its linear velocity tangential to the path.

2) Formation coordination assignment: guarantees synchronization of all the path parameters $\tau_{i}$, such that vehicles in the networked team keep the desired relative distance to the FRV in the formation.

This coordinated path tracking control methodology belongs to the "Divide to Conquer" strategy original proposed for coordinated path following control in [29], [39]. Using this framework, path convergence and inter-vehicle coordination can be essentially divided and decoupled. Path convergence for each vehicle aims at driving the tracking error to zero. Intervehicle coordination is achieved by adapting the speed of each virtual vehicle along its path according to the communicated path parameter $\tau_{i}$ of other virtual vehicles. In this strategy, complicated kinematic or dynamic information is not required to be exchanged among the networked vehicles, as compared to the heavy information exchanges in [40].

Revisiting the path tracking assignment, it means that the path tracking error between the $i$-th physical vehicle and the $i$-th virtual vehicle should be zero. Let the path tracking error vector $p_{e i B}=\left[x_{e i}, y_{e i}, \psi_{e i}\right]^{T}=R_{I}^{B_{i}}\left(\psi_{i}\right)\left(p_{r i}-p_{i}\right)$ be built in the $i$-th vehicle body frame $\left\{B_{i}\right\}$, as illustrated in Fig. 1. There is:

$$
p_{e i B}=\left[\begin{array}{ccc}
\cos \psi_{i} & \sin \psi_{i} & 0 \\
-\sin \psi_{i} & \cos \psi_{i} & 0 \\
0 & 0 & 1
\end{array}\right]\left[\begin{array}{c}
x_{r i}-x_{i} \\
y_{r i}-y_{i} \\
\psi_{r i}-\psi_{i}
\end{array}\right]
$$




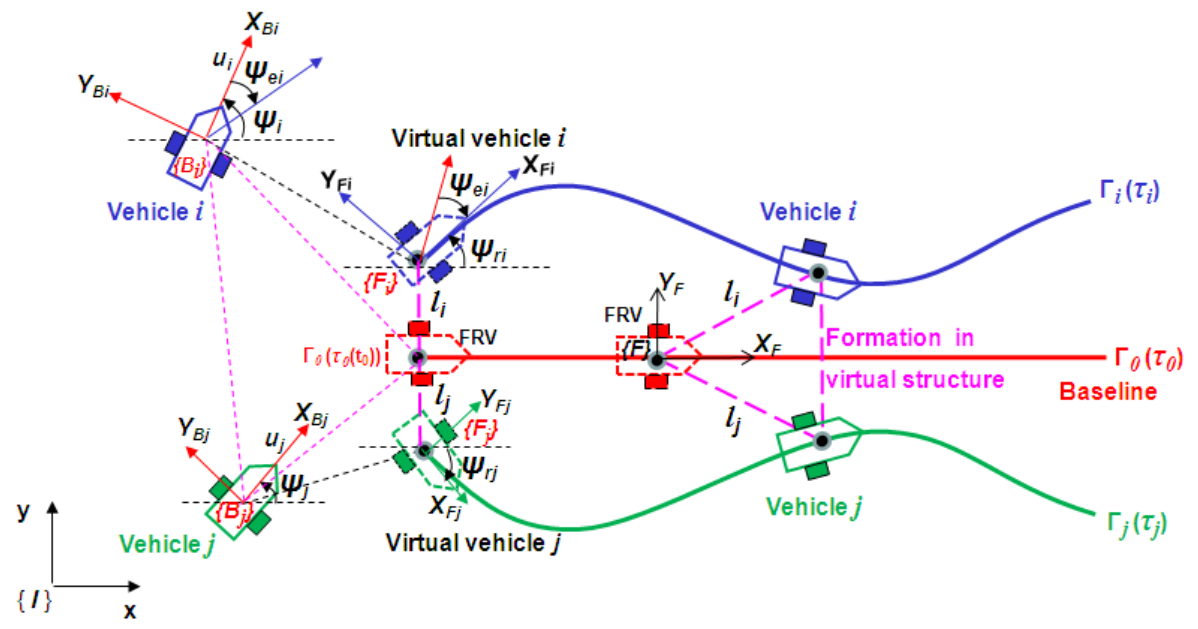

Fig. 2. Paths setup for coordinated formation tracking

where $p_{i}=\left[x_{i}, y_{i}, \psi_{i}\right]^{T}$ is the state vector of the $i$-th physical vehicle, and $p_{r i}=\left[x_{r i}, y_{r i}, \psi_{r i}\right]^{T}$ is the corresponding state vector of the virtual vehicle moving along the $i$-th reference path in the inertial frame $\{I\}$.

Therefore, according to the path tracking and formation coordination assignments declared before, the control objective of coordinated path tracking is to design a controller and achieve the following tasks:

$$
\begin{gathered}
\lim _{t \rightarrow \infty}\left\|p_{e i B}\right\|=0 \\
\lim _{t \rightarrow \infty}\left|\tau_{i}(t)-\tau_{j}(t)\right|=0, \lim _{t \rightarrow \infty}\left|\dot{\tau}_{i}(t)-\dot{\tau}_{0}(t)\right|=0
\end{gathered}
$$

\section{COORDINATED PATH TRACKING CONTROL DESIGN}

\section{A. Error dynamics of path tracking}

Assume that the $i$-th vehicle moves with velocity $q_{i}=$ $\left(u_{i}, \omega_{i}\right)^{T}$, and the $i$-th virtual reference vehicle moves with velocity $q_{r i}=\left(u_{r i}, \omega_{r i}\right)^{T}$. Differentiating the error vector (8), yields error dynamics

$$
\left[\begin{array}{c}
\dot{x}_{e i} \\
\dot{y}_{e i} \\
\dot{\psi}_{e i}
\end{array}\right]=\left[\begin{array}{ccc}
0 & \omega_{i} & 0 \\
-\omega_{i} & 0 & 0 \\
0 & 0 & 0
\end{array}\right] p_{e i B}+R_{I}^{B i}\left(\psi_{i}\right)\left[\begin{array}{c}
\dot{x}_{r i}-\dot{x}_{i} \\
\dot{y}_{r i}-\dot{y}_{i} \\
\dot{\psi}_{r i}-\dot{\psi}_{i}
\end{array}\right]
$$

Note that each virtual vehicle has the same kinematic model with that of the physical vehicle in (1). Substituting (8) into (11), there is

$$
\begin{aligned}
{\left[\begin{array}{c}
\dot{x}_{e i} \\
\dot{y}_{e i} \\
\dot{\psi}_{e i}
\end{array}\right]=} & {\left[\begin{array}{ccc}
0 & \omega_{i} & 0 \\
-\omega_{i} & 0 & 0 \\
0 & 0 & 0
\end{array}\right]\left[\begin{array}{c}
x_{e i} \\
y_{e i} \\
\psi_{e i}
\end{array}\right] } \\
& +R_{I}^{B i}\left(\psi_{i}\right)\left[\begin{array}{c}
u_{r i} \cos \psi_{r i}-u_{i} \cos \psi_{i} \\
u_{r i} \sin \psi_{r i}-u_{i} \sin \psi_{i} \\
\dot{\psi}_{r i}-\dot{\psi}_{i}
\end{array}\right] \\
= & {\left[\begin{array}{c}
\omega_{i} y_{e i}-u_{i}+u_{r i} \cos \left(\psi_{r i}-\psi_{i}\right) \\
-\omega_{i} x_{e i}+u_{r i} \sin \left(\psi_{r i}-\psi_{i}\right) \\
\omega_{r i}-\omega_{i}
\end{array}\right] }
\end{aligned}
$$

As the linear velocity of the $i$-th virtual vehicle is tangential to the $i$-th path $\Gamma_{i}\left(\tau_{i}\right)$, there is

$$
\left\{\begin{array}{l}
u_{r i}\left(\tau_{i}(t)\right)=\left|\dot{p}_{r i}\left(\tau_{i}(t)\right)\right|=\sqrt{\dot{p}_{r i}^{T} \dot{p}_{r i}}:=\bar{u}_{r i}\left(\tau_{i}\right) \dot{\tau}_{i} \\
\omega_{r i}\left(\tau_{i}(t)\right)=\dot{\psi}_{r i}\left(\tau_{i}(t)\right)=\psi_{r i}^{\tau_{i}}\left(\tau_{i}\right) \dot{\tau}_{i}:=\bar{\omega}_{r i}\left(\tau_{i}\right) \dot{\tau}_{i}
\end{array}\right.
$$

where $\bar{u}_{r i}\left(\tau_{i}\right), \bar{\omega}_{r i}\left(\tau_{i}\right)$ are defined as

$$
\left\{\begin{array}{l}
\bar{u}_{r i}\left(\tau_{i}\right)=\sqrt{x_{r i}^{\tau_{i}}\left(\tau_{i}\right)^{2}+y_{r i}^{\tau_{i}}\left(\tau_{i}\right)^{2}} \\
\bar{\omega}_{r i}\left(\tau_{i}\right)=\frac{x_{r i}^{\tau_{i}}\left(\tau_{i}\right) y_{r i}^{2}\left(\tau_{i}\right)-x_{i j}^{2}\left(\tau_{i}\right) y_{r i}^{\tau_{i}}\left(\tau_{i}\right)}{x_{r i}^{\tau_{i}}\left(\tau_{i}\right)^{2}+y_{r i}^{\tau_{i}}\left(\tau_{i}\right)^{2}}
\end{array}\right.
$$

Therefore, the relationship between the desired reference velocity $\left(u_{r i}, \omega_{r i}\right)^{T}$ and the speed of the path parameter $\dot{\tau}_{i}$ is built for each virtual vehicle and the corresponding path. It makes sense to conveniently synchronize the path parameters in the coordinated control stage later. Assume each virtual vehicle has the same kinematics with the physical nonholonomic vehicle. Substituting (13) into (12), the corresponding error state dynamics in the $i$-th vehicle body frame $\left\{B_{i}\right\}$ can be rewritten as follows:

$$
\left[\begin{array}{c}
\dot{x}_{e i} \\
\dot{y}_{e i} \\
\dot{\psi}_{e i}
\end{array}\right]=\left[\begin{array}{c}
\omega_{i} y_{e i}-u_{i}+\bar{u}_{r i} \dot{\tau}_{i} \cos \psi_{e i} \\
-\omega_{i} x_{e i}+\bar{u}_{r i} \dot{\tau}_{i} \sin \psi_{e i} \\
\bar{\omega}_{r i} \dot{\tau}_{i}-\omega_{i}
\end{array}\right]
$$

\section{B. Path tracking control}

Before starting the path tracking control design, the following assumption for each desired spatial reference path is given as:

Assumption 3.1: prescribed paths for mobile vehicles

- Uniqueness

For each value of the path parameter $\tau_{i}$, there exists a unique value of $x_{r}\left(\tau_{i}\right)$ and $y_{r}\left(\tau_{i}\right)$. It means the unique solvability of one path from its parameter.

- Regularity

$$
0<\sqrt{x_{r}^{\tau_{i}}\left(\tau_{i}\right)^{2}+y_{r}^{\tau_{i}}\left(\tau_{i}\right)^{2}}<k
$$

Where $k$ is a bounded positive constant. It means the desired path is regularly parameterized. However, any nonregular path can be split into piecewise regular subpaths. 
- Persistent excitation

$$
\lim _{t \rightarrow \infty} \dot{\tau}_{i}(t) \neq 0
$$

The path parameter is persistently excited, which means the path is endless. Therefore, the path tracking problem will not degenerate into the point stabilization problem [30].

Similarly to the path following control design in [33], [41], an approaching angle $\delta_{i}\left(y_{e i}, \dot{\tau}_{0}\right)$ illustrated in Fig. 1, is introduced as a heading guidance for each vehicle in order to shape the desired orientation during transient path tracking behavior, such that

$$
\left\{\begin{array}{c}
\delta_{i}\left(0, \dot{\tau}_{0}\right)=0 \\
-y_{e i} \dot{\tau}_{0} \sin \delta_{i} \geq 0
\end{array}\right.
$$

According to the rules in [38], the approaching angle can be chosen as a sigmoid function $\delta_{i}\left(y_{e i}, \dot{\tau}_{0}\right)=$ $-\operatorname{sign}\left(\dot{\tau}_{0}\right) \psi_{a} \tanh \left(k_{\delta} y_{e i}\right)$, where $\operatorname{sign}(\cdot)$ is the sign function. Actually, this choice is natural in the sense that the approaching angle $\delta_{i}$ provides an adequate reference heading guidance in order to drive the physical vehicle towards the path (turn right when the vehicle is on the left side of the path, and turn left in the other situation) [32]. However, it raises theoretical difficulties because $\delta_{i}$ is not differentiable with respect to $\dot{\tau}_{0}=0$. In the case of coordinated path following, this problematic situation can be avoided by imposing $\dot{\tau}_{0}>0$, which means that the desired path is generated in a forward direction. This condition is reasonable for most of the coordinated path following applications, for instance, coverage and exploration of interested area using coordinated vehicles along predefined paths in a unidirection. Thus, the approaching angle $\delta_{i}\left(y_{e i}, \dot{\tau}_{0}\right)$ can be proposed as

$$
\delta_{i}\left(y_{e i}, \dot{\tau}_{0}\right)=-\psi_{a} \tanh \left(k_{\delta} y_{e i}\right)
$$

where the shaping coefficients $k_{\delta}>0,0<\psi_{a} \leq \pi / 2$.

In order to evaluate the path tracking error, define the error variable $\tilde{\tau}_{i}$ between the actual path parameter $\tau_{i}$ and the desired path parameter, such that its derivative is:

$$
\dot{\tilde{\tau}}_{i}=\dot{\tau}_{i}-v_{\tau i}\left(t, x_{e i}, y_{e i}, \psi_{e i}\right)
$$

where $\dot{\tilde{\tau}}=\left[\dot{\tilde{\tau}}_{i}\right]_{n \times 1}$ can be considered as the speed disagreement error vector of coordinated formation tracking, and $v_{\tau i}\left(t, x_{e i}, y_{e i}, \psi_{e i}\right)$ is the desired speed for each path parameter $\tau_{i}$. Note that $\dot{\tau}_{0}(t)$ is the desired speed assignment for the FRV moving along the baseline path, where $\dot{\tau}_{0}(t)$ can be left free for a remote operator, or solved as a separate task [42]. Hence, $v_{\tau i}$ can be chosen as a feedback function of $\dot{\tau}_{0}(t)$ [30], [43], or equal to $\dot{\tau}_{0}(t)$ in a simplified manner.

The Control Lyapunov function is selected in a positive definite quadratic form as

$$
V=\frac{1}{2} \sum_{i=1}^{n}\left[x_{e i}^{2}+y_{e i}^{2}+\frac{1}{\gamma}\left(\psi_{e i}-\delta_{i}\right)^{2}\right]
$$

The time derivative of (18) along the solution of (12) is

$$
\begin{aligned}
\dot{V}= & \sum_{i=1}^{n}\left[-x_{e i} u_{i}+u_{r i} x_{e i} \cos \psi_{e i}+u_{r i} y_{e i} \sin \delta_{i}\right. \\
& \left.+\frac{1}{\gamma}\left(\psi_{e i}-\delta_{i}\right)\left(\dot{\psi}_{e i}-\dot{\delta}_{i}+\gamma u_{r i} y_{e i} \frac{\sin \psi_{e i}-\sin \delta_{i}}{\psi_{e i}-\delta_{i}}\right)\right]
\end{aligned}
$$

Substituting (14) and (17) into the above derivative of the Lyapunov control function yields

$$
\begin{aligned}
\dot{V}= & \sum_{i=1}^{n}\left\{x_{e i}\left(\bar{u}_{r i} v_{\tau i} \cos \psi_{e i}-u_{i}\right)+y_{e i} \bar{u}_{r i} v_{\tau i} \sin \delta_{i}\right. \\
& +\dot{\tilde{\tau}}_{i}\left[x_{e i} \bar{u}_{r i} \cos \psi_{e i}+y_{e i} \bar{u}_{r i} \sin \delta_{i}+\frac{1}{\gamma}\left(\psi_{i}-\delta_{i}\right) \bar{\omega}_{r i}\right] \\
& \left.+\frac{1}{\gamma}\left(\psi_{e i}-\delta_{i}\right)\left(\bar{\omega}_{r i} v_{\tau i}-\omega_{i}-\dot{\delta}_{i}+\gamma y_{e i} \bar{u}_{r i} v_{\tau i} \frac{\sin \psi_{e i}-\sin \delta_{i}}{\psi_{e i}-\delta_{i}}\right)\right\}
\end{aligned}
$$

Proposing the control inputs as

$$
\left[\begin{array}{c}
u_{i} \\
\omega_{i}
\end{array}\right]=\left[\begin{array}{l}
k_{e} x_{e i}+\bar{u}_{r i} v_{\tau i} \cos \psi_{e i} \\
\bar{\omega}_{r i} v_{\tau i}-\dot{\delta}_{i}+\gamma y_{e i} \bar{u}_{r i} v_{\tau i} \frac{\sin \psi_{e i}-\sin \delta_{i}}{\psi_{e i}-\delta_{i}}+k_{\psi}\left(\psi_{e i}-\delta_{i}\right)
\end{array}\right]
$$

and directly choosing $v_{\tau i}=\dot{\tau}_{0}$, yields

$$
\dot{V}=-\sum_{i=1}^{n}\left[k_{e} x_{e i}^{2}-\phi_{i} \dot{\tau}_{i}-y_{e i} \bar{u}_{r i} \dot{\tau}_{0} \sin \delta_{i}+\frac{k_{\psi}}{\gamma}\left(\psi_{e i}-\delta_{i}\right)^{2}\right]
$$

where $\phi_{i}:=x_{e i} \bar{u}_{r i} \cos \psi_{e i}+y_{e i} \bar{u}_{r i} \sin \delta_{i}+\frac{1}{\gamma}\left(\psi_{e i}-\delta_{i}\right) \bar{\omega}_{r i}$ for simplified notation.

Furthermore, choosing $\dot{\tilde{\tau}}_{i}=-k_{\tau i} \phi_{i}, k_{\tau i}>0$, drives

$$
\dot{V}=-\sum_{i=1}^{n}\left[k_{\tau i} \phi_{i}^{2}+k_{e} x_{e i}^{2}-y_{e i} \bar{u}_{r i} \dot{\tau}_{0} \sin \delta_{i}+\frac{k_{\psi}}{\gamma}\left(\psi_{e i}-\delta_{i}\right)^{2}\right]
$$

to be negative semidefinite, where $y_{e i} \bar{u}_{r i} \dot{\tau}_{0} \sin \delta_{i} \leq 0$ due to the definition of the approaching angle in (16).

Moreover, it is straightforward to show that $\ddot{V}$ is bounded such that $\dot{V}$ is uniformly continuous. By applying Barbalat's lemma [44], the equation (21) results in $\lim _{t \rightarrow \infty}\left(x_{e i}, y_{e i}, \psi_{e i}\right)^{T}=0^{3}$. Hence, $\lim _{t \rightarrow \infty}\left\|p_{e i B}\right\|=0$. It means each physical vehicle coincides with the corresponding virtual vehicle moving along the prescribed path, such that the first objective in (9) is fullfilled and the path tracking assignment is achieved. The next problem is rested to address the formation coordination assignment in order to achieve the objective in (10).

Note that the term $\frac{\sin \psi_{e i}-\sin \delta_{i}}{\psi_{e}-\delta_{i}}$ of the control law for $\omega_{i}$ in (19) is well defined and continuous at zero. Therefore, the path tracking controller is nonsingular in the whole time horizon.

\section{Formation coordination control}

Apparently, necessary information must be communicated and exchanged among a team of networked vehicles, in order to coordinate the whole team. Therefore, information flow in the communication network must be carefully treated, which plays a key role in decentralized control of networked vehicles. In [45], [46], algebraic graph theory is introduced to represent the communication network, where each vehicle is one node and each communication link is one edge in the graph. 
1) Graph representation of networked vehicles: In this section, the basic concept of graph and matrices associated with graph are reviewed, which are the preliminaries of algebraic graph theory. See for example [47], [48] and the references therein.

A communication topology is defined by a graph $G(V, E)$ with $N$ vertices in a set of vertices $V$, and a set of edges $E$ with edges $e_{i j}=\left(v_{i}, v_{j}\right) \in E$ with $v_{i}, v_{j} \in V$. The set of neighbors of vertex $v_{i}$ is denoted by $J_{i}=\left\{j:\left(v_{i}, v_{j}\right) \in E\right\}$. Vertex $v_{i}$ and $v_{j}$ are adjacent if $\left(v_{i}, v_{j}\right) \in E$. The adjacency matrix $A$ of graph $G$ is a positive square matrix of size $|V|$, whose $i j$ th element $a_{i j}=$ 1 if $\left(v_{i}, v_{j}\right) \in E$, and is zero otherwise. The degree of vertex $v_{i}$ is defined by $\operatorname{deg}\left(v_{i}\right)=\sum_{j=1}^{n} a_{i j}$, and the degree matrix $D=$ $D(A)$ of an undirected graph $G$ is the diagonal matrix with the diagonal element $D_{i i}=\operatorname{deg}\left(v_{i}\right)$. The graph Laplacian matrix $L=\left[l_{i j}\right]$ of an undirected graph is an $n \times n$ matrix associated with graph $G$, defined as $L=D(A)-A$. A path in the graph is a sequence of distinct vertices. A graph is said to be connected if there is a path between any distinct pair of vertices.

Lemma 1: From [48], the Laplacian matrix of a connected graph only has one single zero eigenvalue and the corresponding eigenvector is the vector of ones, $\overrightarrow{1}$.

In this paper, we give the following assumption for the communication topology of networked multi-vehicle systems:

Assumption 3.2: The information exchange between vehicles is bidirectional, and the related undirected graph $G$ of the communication network in consideration is connected.

2) Distributed control of networked vehicles: In order to make the presentation clear, the following vector notions are used: $\Omega_{\tau}=\left[\dot{\tilde{\tau}}_{1}, \ldots, \dot{\tilde{\tau}}_{n}\right]^{T}, \mathscr{T}=\left[\tau_{1}, \ldots, \tau_{n}\right]^{T}$, and $V_{\tau}=$ $\left[v_{\tau 1}, \ldots, v_{\tau n}\right]^{T}$. Thus, (17) can be rewritten as

$$
\dot{\mathscr{T}}=\Omega_{\tau}+V_{\tau}
$$

Augmenting the Lyapunov function (18) as

$$
V_{\text {aug }}=V+\frac{1}{2} \Omega_{\tau}^{T} K_{1}^{-1} K_{2}^{-1} \Omega_{\tau}+\frac{1}{2} \mathscr{T}^{T} L \mathscr{T}
$$

where $L$ is the Laplacian matrix of the connected graph $G$, which describes the inter-vehicle communication topology, $K_{1}, K_{2}$ are diagonal positive-definite matrices.

As the communication graph $G$ is bi-directional and connected, it is declared that $L$ is a symmetric positive semidefinite matrix [48]. There is $\frac{d}{d t}\left(\mathscr{T}^{T} L \mathscr{T} / 2\right)=\mathscr{T}^{T} L \mathscr{T}$. Hence, the time derivative of (23) along the solutions of (13), (14) and (19) gives:

$$
\begin{aligned}
\dot{V}_{a u g}= & \Phi^{T} \Omega_{\tau}-\sum_{i=1}^{n}\left[k_{e} x_{e i}^{2}-y_{e i} \bar{u}_{r i} \dot{\tau}_{0} \sin \delta_{i}+\frac{k_{\psi}}{\gamma}\left(\psi_{e i}-\delta_{i}\right)^{2}\right] \\
& +\dot{\Omega}_{\tau}^{T} K_{1}^{-1} K_{2}^{-1} \Omega_{\tau}+\mathscr{T}^{T} L \dot{\mathscr{T}}
\end{aligned}
$$

where the vector $\Phi=\left[\phi_{1}, \ldots, \phi_{n}\right]^{T}$.

Proposing the feedback law for path parameters

$$
\left[\begin{array}{l}
\dot{T} \\
\dot{\Omega}_{\tau}
\end{array}\right]=\left[\begin{array}{l}
\Omega_{\tau}+V_{\tau} \\
-K_{1} K_{2}(L \mathscr{T}+\Phi)-K_{1} \Omega_{\tau}
\end{array}\right]
$$

where $V_{\tau}=\left[v_{\tau i}\right]_{n \times 1}=\dot{\tau}_{0} \overrightarrow{1}$ with $\overrightarrow{1}=[1]_{n \times 1}$, and $\lim _{t \rightarrow \infty} \dot{\tau}_{0} \neq 0$. It means that the desired time evolving speed $v_{\tau i}$ is chosen as the same for each vehicle, and equals to the desired formation speed $\dot{\tau}_{0}$. Whereas, $\dot{\tau}_{0}$ needs not be constant and it could be time-varying.

Now, the solution to the coordinated path tracking problem can be proposed, with decentralized feedback law for the path parameter vector $\mathscr{T}$ as a function of the information received from the neighboring vehicles in the network.

Theorem 3.3: Decentralized control of coordinated path tracking in kinematics stage

Under assumptions 3.1 and 3.2, the kinematic control input $\left(u_{i}, \omega_{i}\right)^{T}$ and the distributed feedback law for the path parameter $\dot{\tau}_{i}$, are given in (19) and (25) respectively. The control objectives (9) and (10) of coordinated path tracking are achieved, and the equilibrium point $\left[x_{e i}, y_{e i}, \psi_{e i},\left(\tau_{i}-\tau_{j}\right)\right]^{T}=0^{4}$ is globally asymptotically stable.

\section{Remark:}

By examining the compact form of the feedback law proposed in (25), it can be rewritten in a decentralized form as

$$
\left\{\begin{array}{l}
\dot{\tau}_{i}=\dot{\tilde{\tau}}_{i}+v_{\tau_{i}} \\
\ddot{\tilde{\tau}}_{i}=-k_{1 i}\left[\dot{\tilde{\tau}}_{i}+k_{2 i}\left(\sum_{j \in J_{i}}\left(\tau_{i}-\tau_{j}\right)+\phi_{i}\right)\right]
\end{array}\right.
$$

Recall that $J_{i}$ denotes the set of neighboring vehicles (vertices in the communication graph) that communicate with vehicle $i$ in the network. Note that the feedback law of path parameter $\dot{\tau}_{i}$ for vehicle $i$ is a function of its own path parameter errors, with respect to path parameters of other vehicles included in the communication set $J_{i}$. Clearly, the feedback law is decentralized, which meets the constraints imposed by the communication network.

\section{Backstepping dynamics}

The dynamic version of the decentralized control of coordinated path tracking, can be derived by adopting backstepping techniques [44], [49], in terms of the dynamics of the nonholonomic vehicles in (2). Let $\left(u_{i}, \omega_{i}\right)^{T}$ be the virtual control input vector, and $\left(\alpha_{u i}, \alpha_{\omega i}\right)^{T}$ be the corresponding virtual control law. Define the velocity error vector $z_{i}$ as

$$
z_{i}=\left(\begin{array}{c}
z_{u i} \\
z_{\omega i}
\end{array}\right)=\left(\begin{array}{c}
u_{i}-\alpha_{u i} \\
\omega_{i}-\alpha_{\omega i}
\end{array}\right)
$$

Let the Lyapunov function $V_{a u g}$ in (23) be augmented with the quadratic terms of $z_{u i}$ and $z_{r i}$. That is

$$
V_{d y n}=V_{\text {aug }}+\frac{1}{2} \sum_{i=1}^{n} z_{i}^{T} M_{i} z_{i}
$$

where $M_{i}=\left(\begin{array}{cc}m_{i} & 0 \\ 0 & I_{i}\end{array}\right)$, and $m_{i}, I_{i}$ denote the mass and moment of inertia of the $i$-th vehicle. 
The time derivative of $V_{d y n}$ can be written as

$$
\begin{aligned}
& \dot{V}_{d y n}=\Phi^{T} \Omega_{\tau}+\dot{\Omega}_{\tau}^{T} K_{1}^{-1} K_{2}^{-1} \Omega_{\tau}+\mathscr{T}^{T} L \dot{\mathscr{T}} \\
& +\sum_{i=1}^{n}\left\{x_{e i}\left(\bar{u}_{r i} v_{\tau i} \cos \psi_{e i}-z_{u i}-\alpha_{u i}\right)+y_{e i} \bar{u}_{r i} v_{\tau i} \sin \delta_{i}+m_{i} z_{u i} \dot{z}_{u i}\right.
\end{aligned}
$$$$
+\dot{\tilde{\tau}}_{i}\left[x_{e i} \bar{u}_{r i} \cos \psi_{e i}+y_{e i} \bar{u}_{r i} \sin \delta_{i}+\frac{1}{\gamma}\left(\psi_{e i}-\delta_{i}\right) \bar{\omega}_{r i}\right]+I_{i} z_{\omega i} \dot{z}_{\omega i}
$$

$\left.+\frac{1}{\gamma}\left(\psi_{e i}-\delta_{i}\right)\left(\bar{\omega}_{r i} v_{\tau i}-z_{\omega i}-\alpha_{\omega i}-\dot{\delta}_{i}+\gamma y_{e i} \bar{u}_{r i} v_{\tau i} \frac{\sin \psi_{e i}-\sin \delta_{i}}{\psi_{e i}-\delta_{i}}\right)\right\}$

$=\Phi^{T} \Omega_{\tau}+\dot{\Omega}_{\tau}^{T} K_{1}^{-1} K_{2}^{-1} \Omega_{\tau}+\mathscr{T}^{T} L\left(\Omega_{T}+V_{\tau}\right)+\sum_{i=1}^{n}\left\{y_{e i} \bar{u}_{r i} v_{\tau i} \sin \delta_{i}\right.$

$$
+x_{e i}\left(\bar{u}_{r i} v_{\tau i} \cos \psi_{e i}-\alpha_{u i}\right)+z_{\omega i}\left[I_{i} \dot{z}_{\omega i}-\frac{1}{\gamma}\left(\psi_{e i}-\delta_{i}\right)\right]
$$

$+\dot{\tilde{\tau}}_{i}\left[x_{e i} \bar{u}_{r i} \cos \psi_{e i}+y_{e i} \bar{u}_{r i} \sin \delta_{i}+\frac{1}{\gamma}\left(\psi_{e i}-\delta_{i}\right) \bar{\omega}_{r i}\right]+z_{u i}\left(m_{i} \dot{z}_{u i}-x_{e i}\right)$

$\left.+\frac{1}{\gamma}\left(\psi_{e i}-\delta_{i}\right)\left(\bar{\omega}_{r i} v_{\tau i}-\alpha_{\omega i}-\dot{\delta}_{i}+\gamma y_{e i} \bar{u}_{r i} v_{\tau i} \frac{\sin \psi_{e i}-\sin \delta_{i}}{\psi_{e i}-\delta_{i}}\right)\right\}$

Let the virtual control laws $\left.\left(\alpha_{u i}, \alpha_{\omega i}\right)^{T}\right)$ be given according to (19) as follows:

$$
\left[\begin{array}{c}
\alpha_{u i} \\
\alpha_{\omega i}
\end{array}\right]=\left[\begin{array}{l}
k_{e} x_{e i}+\bar{u}_{r i} \dot{\tau}_{0} \cos \psi_{e i} \\
\bar{\omega}_{r i} \dot{\tau}_{0}-\dot{\delta}_{i}+\gamma y_{e i} \bar{u}_{r i} \dot{\tau}_{0} \frac{\sin \psi_{e i}-\sin \delta_{i}}{\psi_{e i}-\delta_{i}}+k_{\psi}\left(\psi_{e i}-\delta_{i}\right)
\end{array}\right]
$$

with $v_{\tau_{i}}=\dot{\tau}_{0}$.

Choose the control law of $\left(F_{i}, N_{i}\right)^{T}$ as

$$
\left\{\begin{array}{l}
F_{i}=m_{i} \dot{u}_{i}=m_{i} \dot{\alpha}_{u i}+x_{e i}-k_{3} z_{u i} \\
N_{i}=I_{i} \dot{\omega}_{i}=I_{i} \dot{\alpha}_{\omega i}+\frac{1}{\gamma}\left(\psi_{e i}-\delta_{i}\right)-k_{4} z_{\omega i}
\end{array}\right.
$$

where $k_{3}, k_{4}$ are positive constants.

Combing (25), (28) and (29), there is

$$
\begin{aligned}
\dot{V}_{d y n} & =-\Omega_{\tau}^{T} K_{2}^{-1} \Omega_{\tau}-\sum_{i=1}^{n}\left[k_{e} x_{e i}^{2}-y_{e i} \bar{u}_{r i} \dot{\tau}_{0} \sin \delta_{i}+\frac{k_{\psi}}{\gamma}\left(\psi_{e i}-\delta_{i}\right)^{2}\right. \\
& \left.+k_{3} z_{u i}^{2}+k_{4} z_{\omega i}^{2}\right] \leq 0
\end{aligned}
$$

That means, $\dot{V}_{d y n}$ is negative semidefinite and all the states $\left(x_{e i}, y_{e i}, \psi_{e i}, z_{u i}, z_{\omega i}\right)$ globally converge to its equilibrium. Moreover, it can be concluded that the equilibrium is $\left(x_{e i}, y_{e i}, \psi_{e i}, z_{u i}, z_{\omega i}\right)^{T}=0^{5}$ from Barbalat's lemma. Therefore, the following proposition can be proposed.

Theorem 3.4: Decentralized control of coordinated path tracking in dynamics stage

Under assumptions 3.1 and 3.2, the dynamics control input $\left(F_{i}, N_{i}\right)^{T}$ and the distributed feedback law for the path parameter $\dot{\tau}_{i}$, are given in (29) and (25) respectively. The control objectives (9) and (10) of coordinated path tracking are achieved, and the equilibrium point $\left[x_{e i}, y_{e i}, \psi_{e i},\left(\tau_{i}-\tau_{j}\right)\right]^{T}=0^{4}$ is globally asymptotically stable.

The proof is omitted due to limited space, which can be completed by using a similar method as in Appendix.

\section{EXAMPLES OF COORDINATED PATH TRACKING CONTROL}

In this section, numerical simulations are carried out in order to illustrate the performance of the proposed coordination control laws for networked nonholonomic mobile vehicles.
1) Circle formation of 10 networked vehicles: In this example, a networked team of $n=10$ identical nonholonomic unicycle-type mobile vehicles undergo path tracking control in circle formation. The physical parameters of the $i$-th vehicle, $i \in\{1, \ldots, n\}$, are taken from [36]:

$$
\left[\begin{array}{l}
m_{i} \\
I_{i}
\end{array}\right]=\left[\begin{array}{l}
9 k g \\
0.1 \mathrm{~kg} \\
m^{2}
\end{array}\right]
$$

The initial velocities are set as $u_{i}=0.2 \mathrm{~m} / \mathrm{s}, \omega_{i}=0 \mathrm{rad} / \mathrm{s}$. The initial positions and orientations of 10 vehicles are as follows:

$\left[\begin{array}{l}x_{i} \\ y_{i} \\ \psi_{i}\end{array}\right]=\left[\begin{array}{l}0,-5,-5,-5,-10,-10,-10,-15,-15,-15 \\ 0,0,5,-5,0,5,-5,0,-5,5 \\ 0,0,0,0,0,0,0,0,0,0\end{array}\right]$

The path parameter of the FRV which generates the baseline for the whole formation is taken as

$$
\Gamma_{0}\left(x_{r 0}, y_{r 0}, \psi_{r 0}\right)=\left[\tau_{0}, 10 \sin \left(0.1 \tau_{0}\right), \operatorname{atan} 2\left(\cos \left(0.1 \tau_{0}\right)\right)\right]^{T}
$$

The offset vector from the virtual vehicles (or virtual targets) to the FRV are defined as

$$
l_{i}\left(\left(x_{r i}\left(\tau_{i}\right), y_{r i}\left(\tau_{i}\right)\right)=\left[\begin{array}{c}
10 \sin (2 \pi(i-1) / N) \\
10 \cos (2 \pi(i-1) / N \\
0
\end{array}\right]\right.
$$

where $i \in\{1,2, \ldots, n\}$. This means that the baseline $\Gamma_{0}$ is a sinusoidal path, and the desired paths for 10 vehicles are generated from the baseline according to (3), (30) and (31). Moreover, the desired virtual structure and formation shape is a circle, as its vertices are uniformly distributed on a circle centered on the sinusoidal baseline with radius of $10 \mathrm{~m}$.

The timing law of the FRV path parameter is set as $\dot{\tau}_{0}(t)=0.1 \mathrm{~m} / \mathrm{s}$. The initial path parameters related to the FRV and virtual vehicles are set as $\tau_{0}=0, \tau_{1}=2, \tau_{2}=0, \tau_{3}=2, \tau_{4}=$ $0, \tau_{5}=2, \tau_{6}=0, \tau_{7}=2, \tau_{8}=0, \tau_{9}=0, \tau_{10}=8$. Furthermore, the velocity limitations of the vehicle, $0<u_{i}(t)<1.2 \mathrm{~m} / \mathrm{s}$ and $-1.5 \mathrm{rad} / \mathrm{s} \leq \omega(t)_{i} \leq 1.5 \pi \mathrm{rad} / \mathrm{s}$, are imposed in the simulation. The control gains are $k_{e}=1, \gamma=2, k_{\psi}=1, \psi_{a}=$ $\pi / 2, k_{1 i}=1, k_{2 i}=1, k_{3}=0.5, k_{4}=1$.

The Laplacian matrix $L$ related to the communication topology of the 10 networked vehicles is defined as follows:

$$
L=\left[\begin{array}{cccccccccc}
3 & -1 & -1 & 0 & 0 & 0 & 0 & 0 & 0 & -1 \\
-1 & 1 & 0 & 0 & 0 & 0 & 0 & 0 & 0 & 0 \\
0 & -1 & 0 & 2 & -1 & 0 & 0 & 0 & 0 & 0 \\
0 & 0 & -1 & 2 & -1 & 0 & 0 & 0 & 0 & 0 \\
0 & 0 & 0 & -1 & 1 & 0 & 0 & 0 & 0 & 0 \\
0 & 0 & 0 & 0 & 0 & 1 & -1 & 0 & 0 & 0 \\
0 & 0 & 0 & 0 & 0 & -1 & 3 & -1 & 0 & -1 \\
0 & 0 & 0 & 0 & 0 & 0 & -1 & 1 & 0 & 0 \\
0 & 0 & 0 & 0 & 0 & 0 & 0 & 0 & 1 & -1 \\
-1 & 0 & 0 & 0 & 0 & 0 & -1 & 0 & -1 & 3
\end{array}\right]
$$

which means the maximum degree of the communication network is 3 .

In Fig. 3(a), all vehicles asymptotically track their reference paths while building the circle formation simultaneously, where the baseline, the reference path (generated by the virtual 


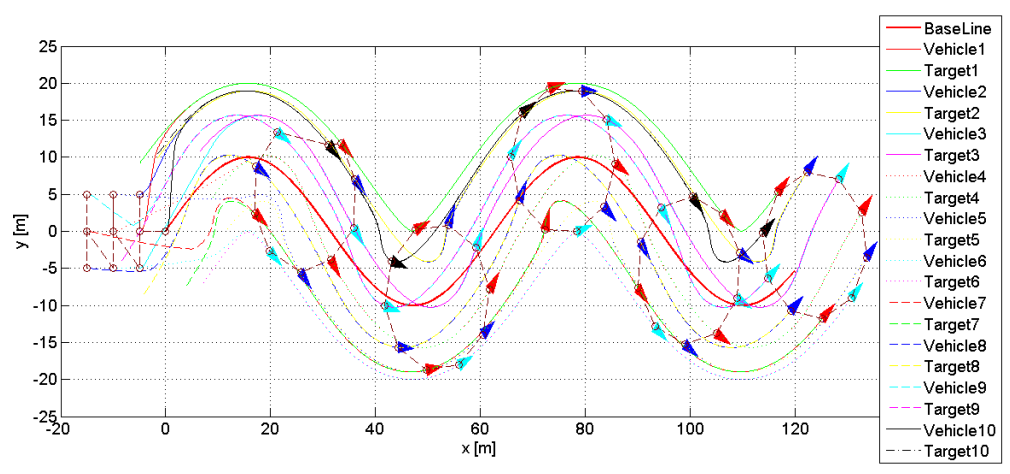

(a) Evolution of building circle formation

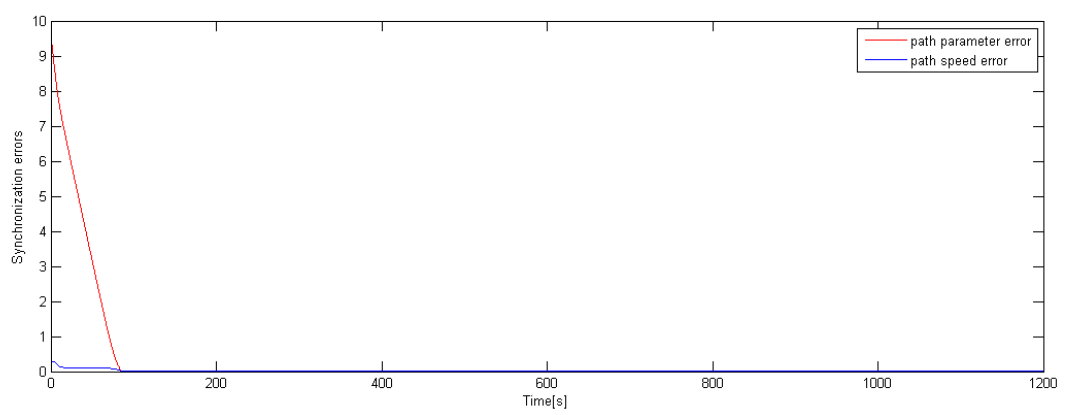

(b) Path synchronization errors: $\sqrt{\sum\left(\tau_{i}-\tau_{j}\right)^{2}}$ and $\sqrt{\sum\left(\dot{\tau}_{i}-\dot{\tau}_{j}\right)^{2}}$

Fig. 3. Circle formation based on coordinated path tracking
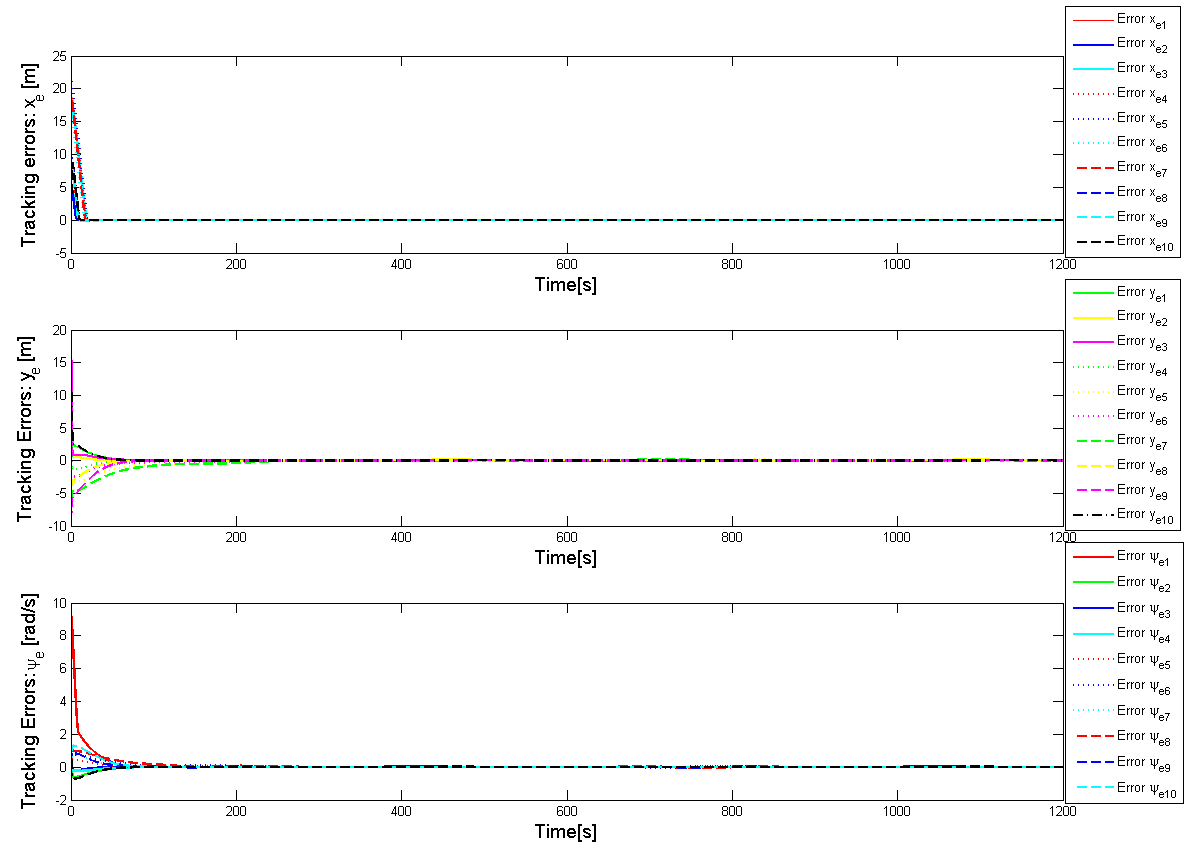

Fig. 4. Path tracking errors: $x_{e i}, y_{e i}, \psi_{e i}$

vehicle/target) and the actual trajectory of the $i$-th physical vehicle are plotted respectively. Note that the orientations of the physical vehicles illustrated by small triangles, are tangential to the sinusoidal paths. In Fig. 3(b), the synchronization errors of the path parameters and their speed disagreement converge to zero, explaining the successful formation coordination.

In Fig. 4, the path tracking errors are plotted, including the position errors $\left(x_{e i}, y_{e i}\right)$ and orientation errors $\psi_{e i}$. The evolving velocities and orientations of the virtual vehicles moving along the paths and those of the actual vehicles are 

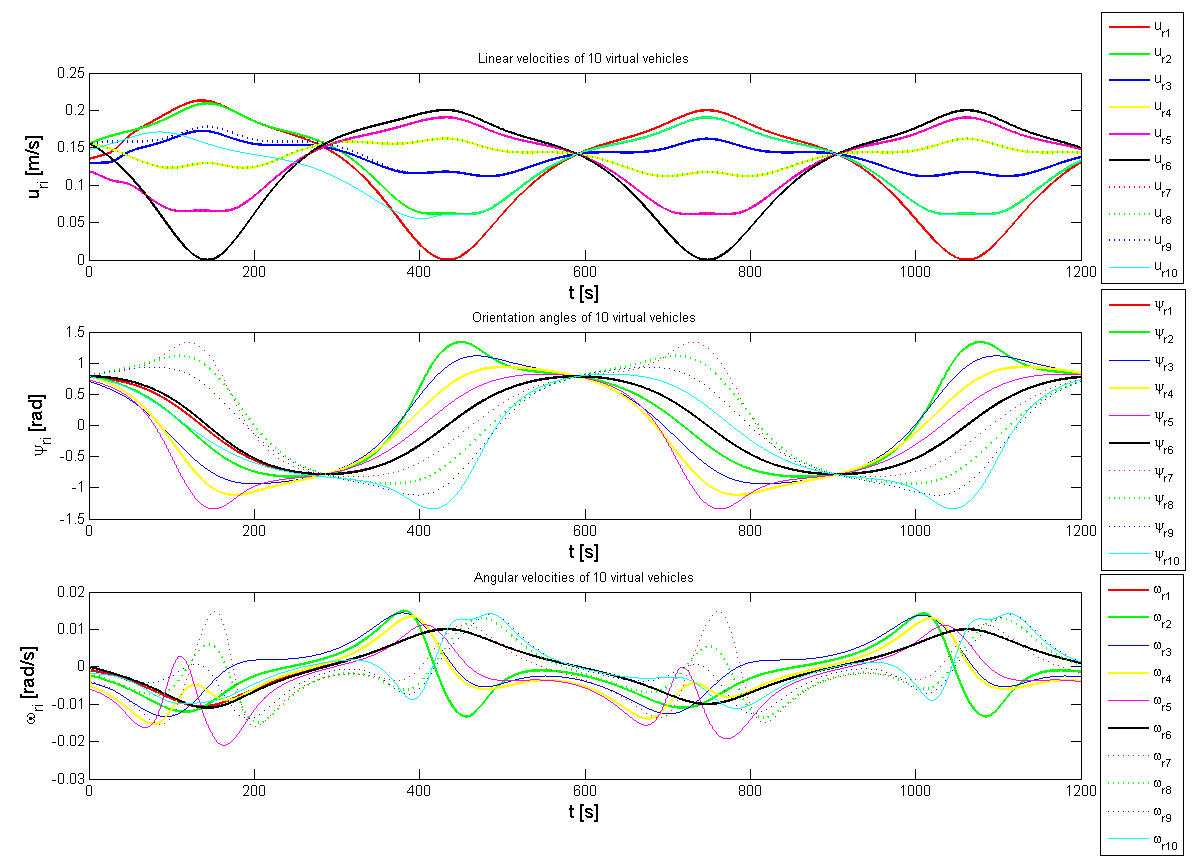

Fig. 5. Time evolution of velocities of 10 virtual vehicles
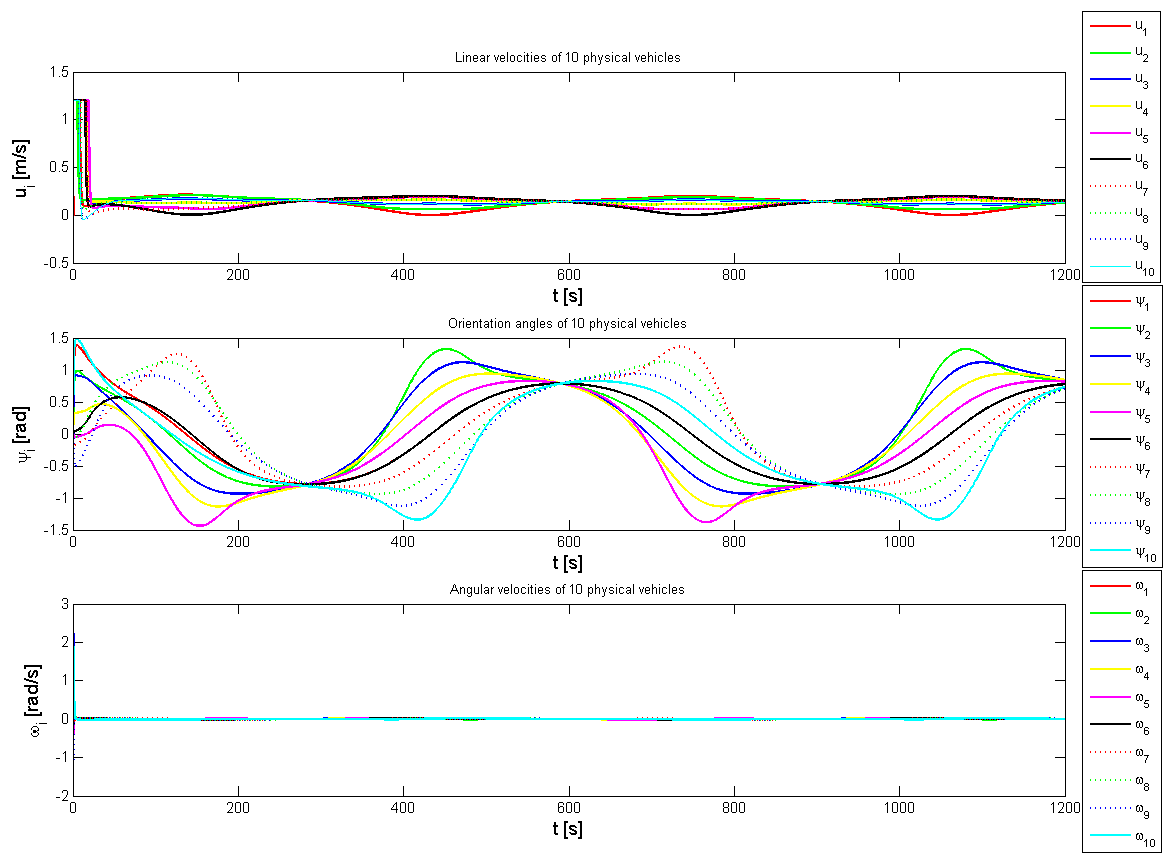

Fig. 6. Time evolution of velocities of 10 physical vehicles while building circle formation

shown in Fig. 5 and Fig. 6 respectively, where the vehicles move with varying velocities along the sinusoidal paths in order to maintain the circle formation. Note that each physical vehicle suffers from limitations on actual linear and angular velocities given in the simulation conditions, so the velocities of physical vehicles are prone to be saturated in the early stage while tracking corresponding virtual vehicles moving along the path. It is clearly interpreted by the differences between the velocities of the virtual vehicles and the physical vehicles shown in the first and third sub-figures in Fig. 5 and Fig. 6, while the orientations of the physical vehicles tend to those of the virtual vehicles quickly, due to the heading guidance. However, the velocities of the physical and virtual vehicles are the same when the physical vehicles catch up with the corresponding virtual vehicles. The vehicles maintain the desired circle formation through the distributed control laws.

2) Scaling circle formation of 10 networked vehicles: In this simulation, the shrunk circle formation is built according 
to the rotation and scaling representation of the virtual structure given in (5). Since the rotation of the circle does not make much sense, only the scaling parameters in (32) are used here to build the shrunk circle formation, defined as

$$
S=\left[\begin{array}{cc}
a\left(1-k \frac{t}{T}\right) & 0 \\
0 & b\left(1-k \frac{t}{T}\right)
\end{array}\right]
$$

where $a=b=1 ; k=0.7, T=1000$. The formation begins shrinking at the time instant $t=600$. Initial conditions are set as the same as the previous example.

The simulation result is given in Fig. 7. It shows that the circle formation is shrinking, and the achieved final circle formation has a radius that is $30 \%$ the size of the original radius at the time instant $t=1000$, as $\left.a\left(1-k \frac{t}{T}\right)\right|_{t=1000}=$ $\left.b\left(1-k \frac{t}{T}\right)\right|_{t=1000}=0.3$.

\section{CONCLUSIONS}

In this paper, the problem of coordinated path tracking for a team of networked nonholonomic mobile vehicles is addressed by modifying the conventional virtual structure approach and combining individual path tracking control design. Through the bi-directional and connected communication network, a distributed feedback law for the path parameters related to the virtual vehicles is derived, such that the desired formation structure is asymptotically built based on graph theory and nonlinear control techniques. Simulation results illustrate the efficiency of the proposed controller. Future work is to extend the techniques developed in this paper combined with matrix decomposition theory to coordinated path tracking under unidirectional communication constraints, and the classical Lyapunov-Krasovskii functional techniques will be resorted for the stability analysis of networked vehicle systems with time varying delays as well. Collision avoidance among vehicles will also be integrated while considering limited sensing ability of the physical vehicles.

\section{ACKNOWLEDGMENT}

The authors would like to thank the anonymous reviewers for their valuable comments and suggestions to improve the quality of this paper.

\section{APPENDIX}

Proof of Theorem 3.3

Given the solution (25), the derivative of Lyapunov candidate function in (24), can be further written as

$$
\begin{aligned}
\dot{V}_{\text {aug }}= & \Phi^{T} \Omega_{\tau}+\dot{\Omega}_{\tau}^{T} K_{1}^{-1} K_{2}^{-1} \Omega_{\tau}+\mathscr{T}^{T} L\left(\Omega_{\tau}+V_{\tau}\right) \\
& -\sum_{i=1}^{n}\left[k_{e} x_{e i}^{2}+y_{e i} \bar{u}_{r i} \dot{\tau}_{0} \sin \delta_{i}+\frac{k_{\psi}}{\gamma}\left(\psi_{e i}-\delta_{i}\right)^{2}\right] \\
= & \left(\Phi^{T}+\dot{\Omega}_{\tau}^{T} K_{1}^{-1} K_{2}^{-1}+\mathscr{T}^{T} L\right) \Omega_{\tau}+\dot{\tau}_{0} \mathscr{T}^{T} L \overrightarrow{1} \\
& -\sum_{i=1}^{n}\left[k_{e} x_{e i}^{2}+y_{e i} \bar{u}_{r i} \dot{\tau}_{0} \sin \delta_{i}+\frac{k_{\psi}}{\gamma}\left(\psi_{e i}-\delta_{i}\right)^{2}\right] \\
= & -\Omega_{\tau}^{T} K_{2}^{-1} \Omega_{\tau}-\sum_{i=1}^{n}\left[k_{e} x_{e i}^{2}-y_{e i} \bar{u}_{r i} \dot{\tau}_{0} \sin \delta_{i}+\frac{k_{\psi}}{\gamma}\left(\psi_{e i}-\delta_{i}\right)^{2}\right]
\end{aligned}
$$$$
\leq 0
$$

where $\dot{\tau}_{0} \mathscr{T}^{T} L \overrightarrow{1}=0$ is applied due to the property of Laplacian matrix $L$ of the connected undirected graph, according to Lemma 1.

Since the control Lyapunov function $V_{\text {aug }}$ is positive definite and radially unbounded from (23), and $\dot{V}_{\text {aug }} \leq 0$, for any initial condition of the state $X=\left(x_{e i}, y_{e i}, \psi_{e i}, \delta_{i}, \Omega_{\tau}\right)^{T}$, there exists a constant $\varepsilon$ such that $\|X(t)\| \leq \varepsilon$ for all $t \geq t_{0}$. Afterwards, tedious but straightforward computation shows that $\ddot{V}_{\text {aug }}\left(t, X_{1}(t)\right)$ exits and is bounded, such that $\dot{V}_{\text {aug }}\left(t, X_{1}(t)\right)$ is uniformly continuous. By using Barbalat's Lemma, we can conclude that

$$
\lim _{t \rightarrow \infty} \dot{V}_{\text {aug }}\left(t, X_{1}(t)\right)=0
$$

Therefore, $\left(x_{e i}, y_{e i}, \psi_{e i}, \delta_{i}, \Omega_{\tau}\right)^{T}$ vanishes as $t \rightarrow \infty$, which drives $\lim _{t \rightarrow \infty} \Phi=0$ and $\lim _{t \rightarrow \infty} \dot{\Omega}_{\tau}=0$. Moreover, by using the fact that $L \mathscr{T}=\dot{\Omega}_{\tau}+\Phi+K \Omega_{\tau}$ from (25), we have $\lim _{t \rightarrow \infty} L \mathscr{T}=0$. Applying Lemma 1 , there is

$$
\lim _{t \rightarrow \infty}\left(\tau_{i}-\tau_{j}\right)=0
$$

Hence, all the path parameters of the reference paths are asymptotically synchronized.

Furthermore, $\lim _{t \rightarrow \infty} \Omega_{\tau}=0$ drives $\lim _{t \rightarrow \infty} \dot{\tilde{\tau}}_{i}=0$. As $\dot{\tilde{\tau}}_{i}=$ $\dot{\tau}_{i}-v_{\tau i}$ with $v_{\tau i}=\dot{\tau}_{0}$ chosen in the control design, there is

$$
\lim _{t \rightarrow \infty}\left|\dot{\tau}_{i}(t)-\dot{\tau}_{0}(t)\right|=0
$$

It means that each nonholonomic mobile vehicle eventually moves along the path according to the desired speed assignment $\dot{\tau}_{0}(t)$. Therefore, the proof is completed.

\section{REFERENCES}

[1] R. M. Murray, "Recent research in cooperative control of multivehicle systems," J. Dyn. Sys., Meas., Controll, vol. 129, no. 5, pp. 571-583, 2007.

[2] P. Antsaklis and J. Baillieul, "Special issue on technology of networked control systems," Proc. of the IEEE, vol. 95, no. 1, pp. 5-8, 2007.

[3] K. Y. Pettersen, J. T. Gravdahl, and H. Nijmeijer, eds., Group Coordination and Cooperative Control, vol. 336 of Lecture Notes in Control and Information Sciences. Heidelberg, Germany: Springer-Verlag, 2006.

[4] F. Bullo, J. Cortes, and B. Piccoli, "Special issue on control and optimization in cooperative networks," SIAM Journal on Control and Optimization, vol. 48, no. 1, pp. vii-vii, 2009.

[5] P. K. C. Wang, "Navigation strategies for multiple autonomous mobile robots moving in formation," J. Robotic Syst., vol. 8, no. 2, pp. 177-195, 1991.

[6] J. P. Desai, J. Ostrowski, and V. Kumar, "Controlling formations of multiple mobile robots," in Proc. IEEE Int. Conf. on Robot. Autom., vol. 4, pp. 2864-2869, 1998.

[7] D. Gu and H. Hu, "A model predictive controller for robots to follow a virtual leader," Robotica, vol. 27, no. 6, pp. 905-913, 2009.

[8] E. Kyrkjebø, K. Y. Pettersen, M. Wondergem, and H. Nijmeijer, "Output synchronization control of ship replenishment operations: Theory and experiments," Control Engineering Practice, vol. 15, no. 6, pp. 741755, 2007.

[9] T. Balch and R. C. Arkin, "Behavior-based formation control for multirobot teams," IEEE Trans. Robot. Automat, vol. 14, no. 6, pp. 926939, 1998.

[10] M. R. Anderson and A. C. Robbins, "Formation flight as a cooperative game," in Proceedings of AIAA Guidance, Navigation and Control Conference, vol. 25, pp. 244-251, 1998.

[11] F. Arrichiello, S. Chiaverini, and T. I. Fossen, "Formation control of underactuated surface vessels using the null-space-based behavioral control," in Proc. IEEE Int. Conf. on Robot. Autom., pp. 5942-5947, 2006.

[12] M. A. Lewis and K. Tan, "High precision formation control of mobile robots using virtual structures," Autonomous Robots, vol. 4, no. 4, pp. 387-403, 1997. 


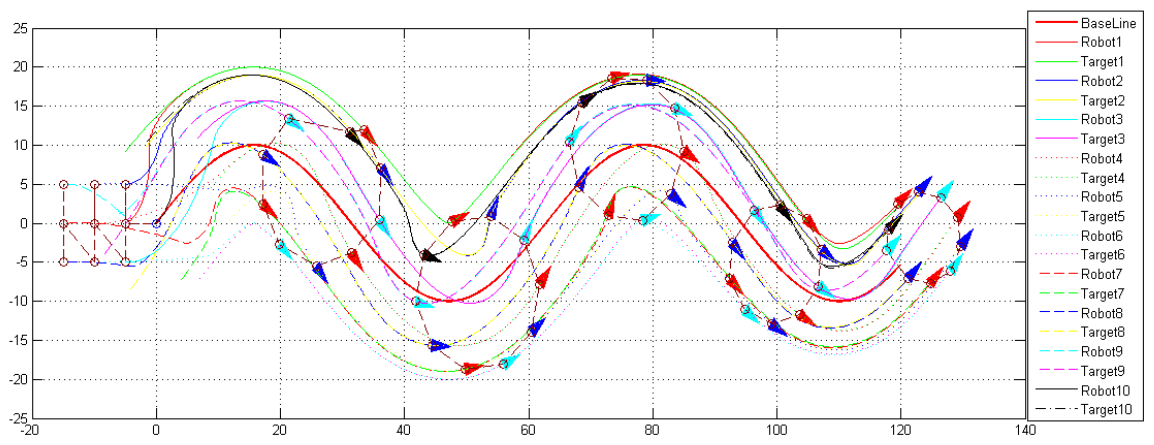

Fig. 7. Shrunk circle formation based on coordinated path tracking

[13] W. Kang and H. Yeh, "Co-ordinated attitude control of multi-satellite systems," Int. J. Robust Nonlinear Control, vol. 12, no. 2-3, pp. 185-205, 2002.

[14] M. Egerstedt and X. Hu, "Formation constrained multi-agent control," IEEE Trans. Robot. Automat, vol. 17, no. 6, pp. 947-951, 2001.

[15] R. Skjetne, S. Moi, and T. Fossen, "Nonlinear formation control of marine craft," in Proc. 41st IEEE Conf. Decision and Control, pp. 16991704, 2002.

[16] K. D. Do, "Formation tracking control of unicycle-type mobile robots," in Proc. IEEE Int. Conf. on Robot. Autom., pp. 2391-2396, 2007.

[17] P. Ogren, E. Fiorelli, and N. E. Leonard, "Cooperative control of mobile sensor networks:adaptive gradient climbing in a distributed environment," IEEE Trans. Autom. Control, vol. 49, no. 8, pp. 1292 1302,2004

[18] E. Fiorelli, N. E. Leonard, P. Bhatta, D. A. Paley, R. Bachmayer, and D. M. Fratantoni, "Multi-auv control and adaptive sampling in monterey bay," IEEE J. Ocean. Eng., vol. 31, no. 4, pp. 935-948, 2006.

[19] R. Olfati-Saber, "Flocking for multi-agent dynamic systems: algorithms and theory," IEEE Trans. Autom. Control, vol. 51, no. 3, pp. 401-420, 2006.

[20] C. C. Cheah, S. P. Hou, and J. J. E. Slotine, "Region-based shape control for a swarm of robots," Automatica, vol. 45, pp. 2406-2411, 2009.

[21] A. Jadbabaie, J. Lin, and A. Morse, "Coordination of groups of mobile autonomous agents using nearest neighbor rules," IEEE Trans. Autom. Control, vol. 48, no. 6, pp. 988-1001, 2003.

[22] Z. Lin, M. Broucke, and B. Francis, "Local control strategies for groups of mobile autonomous agents," IEEE Trans. Autom. Control, vol. 49, no. 4, pp. 622-629, 2004.

[23] H. G. Tanner, A. Jadbabaie, and G. J. Pappas, "Flocking in fixed and switching networks," IEEE Trans. Autom. Control, vol. 52, no. 5, pp. 863-868, 2007.

[24] W. Ren, "On consensus algorithms for double-integrator dynamics," IEEE Trans. Autom. Control, vol. 53, no. 6, pp. 1503-1509, 2008.

[25] I. A. F. Ihle, M. Arcak, and T. I. Fossen, "Passivity-based designs for synchronized path-following," Automatica, vol. 43, pp. 1508-1518, 2007.

[26] L. Lapierre and D. Soetanto, "Coordinated motion control of marine robots," in Proc. 6th Conference on Maneuvering and Control of Marine Craft, 2003.

[27] R. Ghabcheloo, A. Pascoal, C. Silvestre, and I. Kaminer, "Coordinated path following control of multiple wheeled robots using linearization techniques," International Journal of Systems Science, vol. 37, no. 6, pp. 399-414, 2006.

[28] Y. Lan, G. Yan, and Z. Lin, "Synthesis of distributed control of coordinated path following based on hybrid approach," IEEE Trans. Automat. Contr., vol. 56, no. 5, pp. 1170-1175, 2011.

[29] R. Ghabcheloo, A. Pascoal, C. Silvestre, and I. Kaminer,"Non-linear co-ordinated path following control of multiple wheeled robots with bidirectional communication constraints," Int. J. Adapt. Control Signal Process., vol. 21, no. 2-3, pp. 133-157, 2007.

[30] K. D. Do and J. Pan, "Nonlinear formation control of unicycle-type mobile robots," Robot. Auton. Syst., vol. 55, no. 3, pp. 191-204, 2007.

[31] J. Ghommam, H. Mehrjerdi, M. Saad, and F. Mnif, "Formation path following control of unicycle-type mobile robots," Robot. Auton. Syst., vol. 58, no. 5, pp. 727-736, 2010.

[32] L. Lapierre, R. Zapata, and P. Lepinay, "Combined path-following and obstacle avoidance control of a wheeled robot," Int. J. Robot. Res., vol. 26, no. 4, pp. 361-375, 2007.

[33] L. Lapierre and D. Soetanto, "Nonlinear path-following control of an auv," Ocean Engineering, vol. 34, no. 11-12, pp. 1734 -1744, 2007.

[34] X. Xiang, L. Lapierre, C. Liu, and B. Jouvencel, "Path tracking: Combined path following and trajectory tracking for autonomous underwater vehicles, " in Proc. IEEE/RSJ Int. Conf. on Intelligent Robot and Systems, pp. 3558-3563, 2011.

[35] A. P. Aguiar, Nonlinear Motion Control of Nonholonomic and Underactuated Systems. PhD thesis, IST, Portugal, 2001.

[36] L. Lapierre, D. Soetano, and A. Pascoal, "Nonsingular path following control of a unicycle in the presence of parametric modelling uncertainties," Int. J. Robust Nonlin., vol. 16, no. 10, pp. 485-503, 2006.

[37] G. Oriolo, A. D. Luca, and M. Vendittelli, "WMR control via dynamic feedback linearization: design, implementation, and experimental validation," IEEE Trans. Control Syst. Technol., vol. 10, no. 6, pp. 835-852, 2002.

[38] A. Micaelli and C. Samson, "Trajectory tracking for unicycle-type and two-steering-wheels mobile robots," Research Report RR-2097, INRIA, France, 1993.

[39] J. Almeida, C. Silvestre, and A. Pascoal, "Cooperative control of multiple surface vessels in the presence of ocean currents and parametric model uncertainty," Int. J. Robust Nonlinear Control, vol. 20, no. 14, pp. 1549-1565, 2010.

[40] W. Ren, R. W. Beard, and A. W. Beard, "Decentralized scheme for spacecraft formation flying via the virtual structure approach," AIAA J. Guid. Control. Dynam., vol. 27, pp. 73-82, 2004.

[41] I. Kaminer, A. Pascoal, E. Xargay, N. Hovakimyan, and V. Dobrokhodov, "3D path following for small uavs using commercial autopilots augmented by L1 adaptive control," AIAA J. Guid. Control. Dynam., vol. 33, no. 2, pp. 550-564, 2010.

[42] R. Skjetne, T. I. Fossen, and P. V. Kokotovic, "Robust output maneuvering for a class of nonlinear systems," Automatica, vol. 40, no. 3, pp. 373-383, 2004.

[43] P. Ogren, M. Egerstedt, and X. Hu, "A control lyapunov function approach to multiagent coordination," IEEE Trans. Robot. Automat, vol. 18 , no. 5 , pp. $847-851,2002$.

[44] H. K. Khalil, Nonlinear Systems. Upper Saddle River, NJ: Prentice-Hall, 3rd ed., 2002.

[45] J. A. Fax and R. M. Murray, "Information flow and cooperative control of vehicle formations," IEEE Trans. Autom. Control, vol. 49, no. 9, pp. 1465-1476, 2004.

[46] R. Olfati-Saber and R. M. Murray, "Consensus problems in networks of agents with switching topology and time-delays," IEEE Trans. Autom. Control, vol. 49, no. 9, pp. 1520-1533, 2004.

[47] R. Diestel, Graph Theory. Graduate Texts in Mathematics, New York: Springer-Verlag, 2000.

[48] C. Godsil and G. Royle, Algebraic Graph Theory. Berlin, Germany: Springer-Verlag, 2001.

[49] M. Krstic, I. Kanellakopoulos, and P. Kokotovic, Nonlinear and Adaptive Control Design. New York: Wiley, 1995. 